\title{
Hechos que posicionaron el juego como objeto de estudio. Rastreo a partir de producciones bibliográficas
}

Factsthatpositioned game as anobjectofstudy. Trackingfrombibliographicalproductions

Ivana Verónica Rivero

CONICET-Universidad Nacional de Rio Cuarto, Argentina

ivrivero13@gmail.com

\section{Resumen:}

Juego es un concepto presente en la escritura académica de distintos tiempos que ha sido abordado por autores de diferentes campos de conocimiento. Así, al indagar acerca de los estudios sobre el juego se hace visible un amplio espectro de producciones académicas con distintos grados de formalización (escritos, ensayos, teorías) que le reconocen distintos atributos y le asignan diferentes usos, al punto de que parecieran no hacer referencia al mismo objeto.

Este artículo presenta avances de una investigación que tiene por objetivo conocer y comprender prácticas y discursos acerca del juego en el contexto universitario. Se presenta un relevamiento en la biblioteca de la universidad seleccionada como caso a estudiar, analizando las significaciones que el concepto juego gana en los libros cuyo título lo mencionan. En un primer momento se procedió a un análisis bibliométrico registrando las producciones que lo mencionan y agrupándolas por grandes áreas de conocimiento. En un segundo momento, se seleccionan intencionalmente los libros representativos de distintas líneas de estudio recuperando el contexto en que fueron producidos para reconocer los hechos que posicionaron el juego como objeto de estudio dando origen a discursos incorporados que se intentarán desgravar.

Palabras clave: Juego, Objeto de estudio, Producción bibliográfica, Hechos.

\section{Abstract:}

Game is a concept present in academic writing from different periods and addressed by authors from different fields of knowledge. Thus, when inquiring about the studies on game, it becomes clear that there is a wide spectrum of academic productions with different degrees of formalization (writings, essays, theories) that recognizes several values and assign to it a wide variety of uses, to the extent that they even seem to make reference to different concepts. This article presents advances in research that aim at knowing and understanding practices and discourses about game in the university context. A survey is presented in the library of the university chosen as a case study, analyzing the meanings that the game concept gains in books whose titles mention it. At first, a bibliometric analysis was carried out recording the productions that mention it and grouping them into large areas of knowledge. At a second stage, representative books of different lines of study are intentionally selected, recovering the context in which they were produced to recognize the facts that positioned game as an object of study, giving rise to built-in speeches that we will attempt to deduct.

KEYWORDS: Game, Objectofstudy, Bibliographicalproduction, Facts.

\section{CONTEXTUALIZANDO EL ESCRITO}

Llegué tarde al II Coloquio de la Red Producción (Científica) de las Universidades en el campo de la educación física y la educación de los cuerpos, financiada por la convocatoria del Núcleo de Estudios e Investigaciones en Educación Superior de la Secretaría de Políticas Universitarias Argentina. Fui invitada para presentar en el panel Objeto de estudio.

No solo entré al aula cuando ya había comenzado el encuentro, sino que recién ahora puedo formular mi presentación, pues ha cambiado mi condición laboral. El contar con una beca en Conicet ${ }^{1}$, organismo dedicado a la promoción de la ciencia y la tecnología argentinas, me ha permitido disponer del tiempo

\section{Recepción: 23 de septiembre de 2019 | Aprobación: 28 de mayo de 2020 | Publicación: 01 de junio de 2020}

Cita sugerida: Rivero, I. V. (2020). Hechos que posicionaron el juego como objeto de estudio. Rastreo a partir de producciones bibliográficas. Educación Física y Ciencia, 22(2), e123. https://doi.org/10.24215/23142561e123 
necesario para leer, pensar, madurar ideas y escribir. Tiempo que en el cotidiano ejercicio de la docencia universitaria queda subsumido a resolver cuestiones inmediatas instaladas por la rutina académica y administrativa.

Entonces, se presentan aquí avances de una investigación cualitativa que tiene por objetivo conocer y comprender prácticas y discursos acerca del juego en propuestas pedagógicas del contexto universitario.

El escrito se propone identificar hechos académicos del siglo XX que implicaron una ruptura, un quiebre en el modo de ver, pensar, hacer y decir el juego en el contexto universitario. Hechos que posicionaron el juego como objeto de estudio y pronunciaron discursos sobre juego que encuentran expresión en las prácticas académicas.

La universidad seleccionada intencionalmente como caso a estudiar (UNRC) tiene en su campus una amplia e iluminada biblioteca que mantiene la vigencia y actualización de libros disponibles por pedido de los docentes a cargo de los distintos espacios curriculares. Esta particularidad habilita la opción de realizar un análisis bibliométrico que permita identificar hechos académicos que colocaron los discursos sobre juego que circulan y sostienen prácticas en el contexto universitario actual. Ahora bien, ¿cuál sería el sentido de la búsqueda?

\section{SENTIDO DE LA BÚSQUEDA}

Dice Bourdieu que "hay que buscar en el objeto construido por la ciencia las condiciones sociales de posibilidad del 'sujeto'... a cualquier avance en el conocimiento de las condiciones sociales de producción de los 'sujetos' científicos, corresponde un progreso en el conocimiento del objeto científico, y viceversa" (Bourdieu y Waquant, 2005, p.156).

El juego ha sido un tema constante en el campo de la educación física. Su presencia puede ser rastreada no solo en la producción académica que ha circulado en los primeros años de la educación física universitaria argentina ${ }^{2}$ sino también en las prácticas de intervención docente que dan razón al campo. Tanto en el plano académico como en las prácticas de intervención docente, se puede rastrear que la presencia del juego no escapó a la tendencia marcada por el predominio de argumentos provenientes de un discurso educativo que lo colocó como recurso didáctico para la enseñanza de la gimnasia y el deporte (Rivero, 2011). Esos libros, entre otros, dan cuenta no solo de la presencia del juego en el hacer del profesional de la Educación Física sino del uso del juego como medio para enseñanza de otros saberes. Fue el libro Educación Física Infantil y su didáctica (1997) de González de Alvarez y Rada de Rey, el que se permite sospechar de un uso, otro (al que llaman jugar por jugar), del juego de cara a los desafíos didácticos de la educación física para niños y niñas.

Así, la formación universitaria en educación física hasta fines del siglo pasado en Argentina, presentaba al juego como un medio para la enseñanza de la gimnasia y el deporte. Recién en 1998, el profesor Centurión se hacía responsable de la asignatura Conocimiento y Juego en la Universidad Nacional de Río Cuarto, primera asignaturaque toma al juego como objeto de enseñanza en la educación física universitaria argentina ${ }^{3}$. En el dictado de clases semanales, ensamblaba el juego al tratamiento de las Ciencias Sociales incorporando textos como Homo ludens de Johan Huizinga, Los juegos y los hombres. Las máscaras y el vértigo de Roger Caillois, Fundamentos sociales de la educación física de Celeste Ulrich, El juego y el deporte en el otro generalizado, capítulo del libro Espiritu, persona y sociedad de George Mead. Al mismo tiempo, en la asignatura Sociología de la Educación de la Licenciatura Extraordinaria en Educación Física ${ }^{4}$ presentaba la negociación en el juego, apartado del libroJuegos que vienen de antes. Incorporando el patio a la pedagogía de Víctor Pavía, y el apartado De la regla a la estrategia en Cosas Dichas de Pierre Bourdieu.

Así, se salía del molde hegemónico construido para el estudio del juego en la educación, escapando de no volver al molde propuesto por la medicina y la fisiología, que en el campo de la educación física siempre reclama su territorio. 
Así, se anudaba el estudio del juego a las ciencias sociales en la formación universitaria de educación física. Juego y sociedad un binomio que desde la formación docente puso en escena la acción de jugar (Rivero, 2009) como tesis doctoral (Rivero, 2011). Hizo un aporte al estudio del Jugar de un modo lúdico (Pavía, 2006), desplazó la perspectiva del jugador, que arrincona la densidad teórica en la individualidad, para colocarlo en la perspectiva de los jugadores, mostrando la relevancia del lenguaje en la construcción del juego (Rivero, 2006, Rivero 2009). A partir del uso del lenguaje, se identificaron procesos sociales asociados al estar jugando, la sorpresa y el desafío para conseguir intensidad, la transgresión para sostener la tensión, la relevancia de la risa, el grito y la soltura de movimiento (Rivero, 2011), expresiones rudimentarias poco frecuentes en la escuela (Larrosa, 2000).

La colocación del juego como propuesta académica (Centurión, 2006), la aparición del juego en los NAP (Núcleo de Aprendizaje Prioritario) en 1993 y el consecuente derrame en los diseños curriculares de las distintas provincias ${ }^{5}$, los congresos de Juego en Merlo (Provincia de San Luis) organizados por el Instituto Monseñor Orsali, las mesas alusivas y conferencias en el Congreso Argentino y Latinoamericano de la UNLP, en las Jornadas de Investigación en la UNRC, los trabajos finales de licenciatura en Educación Física y en Nivel Inicial, la construcción de doce tesis de posgrado sobre juego de docentes universitarios de educación física (dos de ellas a nivel de doctorado), fueron construyeron un escenario académico propicio para la producción y discusión sobre el juego en la formación docente, un escenario que sostiene un amplio espectro de prácticas de juego presentes en las aulas, aunque no siempre dichas (Rivero, Gilleta y Limbrici Dagfal, 2019).

Entonces, no es casual que haya elegido el juego para reconocer su configuración como objeto de estudio a partir de discursos que conforman una plurivocalidad simultánea y autoexcluyente que se disputa el universo de las prácticas. El juego es el tema que decidí estudiar desde tuve la oportunidad de elegir. Es el objeto de estudio al que me dedico. Lo he buscado siempre en relación a las intervenciones del docente de Educación Física. La mirada construida no es ingenua, está atravesada por las discusiones académicas que los textos de Bracht en 1996 Educación Física y aprendizaje social, Educação Física e Ciência: cenas de um casamento (in)feliz (1999) han abierto en el campo de conocimiento de procedencia ${ }^{6}$, y por la decisión de sacar el juego de la individualidad para abrazar el arraigambre social y cultural, decisión aprendida en la cultura institucional trazada por las ideas de Centurión en Educación Física y Universidad. Un camino entre el oficio y la profesión (1998).

La incorporación del juego a la formación universitaria supuso un dislocamiento de discursos incorporados que lo desencaja de su función de medio para engranarlo a un conjunto de producciones acerca de la educación del cuerpo que se ofrece propicio para acogerlo objetivado. "Es preciso, entonces, interrogar las prácticas, situar la investigación en un camino que, como la carta robada en el cuento de Poe, sólo porque está inmediatamente ante nosotros resulta difícil hallar" (Crisorio, 2003, p. 24).

\section{LIBROS SOBRE JUEGO}

Juego es un concepto presente en la escritura académica de distintos tiempos que ha sido abordado por autores de diferentes campos de conocimiento. Se puede rastrear la presencia del juego en escritos académicos desde tiempos remotos. Pensadores griegos (como Heráclito), filósofos (Aristóteles, Platón, Schiller, Nieztsche, Wittgenstein, Derrida), pedagogos (Froebel, Montessori), matemáticos (Nash, Morgensten), biólogos (Bally, Maturana-Verden), historiadores (Huizinga), lingistas (Searle), psicólogos (Winnicot, Piaget, Vigotsky, Bruner, Chartier), psiquiatras (Terr), psicomotricistas (Calmels), sociólogos (Bourdieu), antropólogos (Caillois), escribieron sobre el juego. Así, al indagar acerca de los estudios sobre el juego se hace visible un amplio espectro de producciones con distintos grados de formalización (escritos, ensayos, teorías) que le reconocen distintos atributos y le asignan diferentes usos, al punto de que parecieran no hacer referencia al mismo objeto. 
En esta dirección, surgen preguntas como: ¿qué protagonismo gana el juego en esos escritos?, ¿con qué intención los autores escriben sobre juego?, ¿cuáles de los escritos sobre juego son los que aportan a su estudio y no al estudio de otro objeto que el juego pone en escena?, ¿qué atributos son puestos en valor en la mención del juego en esos escritos?, ¿con que universo de palabras se vincula el juego en el marco de esos escritos?, ¿qué hechos académicos colocaron las ideas de juego presentes en esos escritos?, ¿cuáles fueron los contextos de emisión?

Estos interrogantes son ambiciosos, de hecho exceden en sobra este escrito que apela a un análisis mixto que articula componentes cuantitativos y cualitativos, con la intención de encontrar un ordenamiento de la producción académica sobre juego y sus particularidades (como formación de los autores, procedencia, contexto de emisión, continuidad de la línea) que permitan identificar hechos académicos de alta relevancia para el estudio del juego. Cabe aclarar que la investigación en curso hace un rastreo por la presencia del juego en el campo académico que ha llevado a reconocer la amplia paleta de autores que han escrito sobre juego, sin embargo, para este escrito solo se presenta el rastreo de libros que mencionan el juego en su título.

Así, se realizó en un primer momento, un análisis bibliométrico registrando las producciones bibliográficas en cuyo título mencionan el juego ${ }^{7}$, disponibles en la biblioteca de la universidad seleccionada como caso a estudiar, universidad que ofrece tres espacios curriculares de grado, y quince seminarios de posgrado sobre juego ${ }^{8}$ que solicitan la compra de bibliografía fundante que esté disponible en la biblioteca para la consulta.

En un segundo momento, se seleccionan intencionalmente los libros representativos de distintas líneas de estudio recuperando el contexto en que fueron producidos para reconocer los hechos que posicionaron el juego como objeto de estudio dando origen a discursos incorporados que se intentarán desgravar.

La bibliometría pone la atención en productos del pensamiento representados físicamente en los documentos, implica la aplicación de tratamientos cuantitativos a las propiedades del discurso escrito a fin de analizar el curso de la comunicación escrita de una disciplina (Spinak en Montilla Peña, 2012).

Se registraron noventa y ocho libros disponibles en la biblioteca de la Universidad Nacional de Río Cuarto que nombran en su título al menos una de las siguientes palabras: juego, juegos, juguete, jugadores. El indicador de producción permite identificar la actividad académica, en este caso vinculada al juego, pero no necesariamente implica progreso científico, pues las distintas producciones pueden aportar a diferentes líneas de conocimiento. Precisamente por esta razón, los libros seleccionados fueron agrupados en grandes familias detrás de la cuál es factible leer argumentos comunes. Así, los libros fueron organizados en seis categorías según el campo de conocimiento al que aportan: libros de juego que aportan a discusiones del campo de la filosofía, de las ciencias sociales, de la historia, de las ciencias económicas, y de las humanidades. Esta última categoría agrupa la mayor cantidad de libros. Al leer los cincuenta y siete títulos incluidos en el gran apartado humanidades se hizo necesario identificar palabras que permitieran agruparlos en universos de sentido, en definitiva, los campos de conocimiento en los que impactan como producción académica. Fue así que se reconocieron libros que aportan al estudio del deporte, la recreación y la educación física (llamada Humanidades 1 en la Tabla 1), y libros que aportan información sobre distintas poblaciones, franjas etarias y didácticas (llamada Humanidades 2 en Tabla 1). 
TABLA 1

Organización de los libros en función de la palabra mencionada y el campo al que aporta

\begin{tabular}{|c|c|c|c|c|}
\hline & JUEGO & JUEGOS & JUGUETE & JUGADORES \\
\hline $\begin{array}{c}\text { FILOSOFÍA } \\
9 \text { libros en total }\end{array}$ & $\begin{array}{l}\text { Total: } 6 \text { libros } \\
\text { Chartier-El juego } \\
\text { de las reglas } \\
\text { Jacob. El juego de } \\
\text { lo posible } \\
\text { Taylor- El juego } \\
\text { del CI } \\
\text { Winnicot. } \\
\text { Realidady juego } \\
\text { Bottini y Solzi. El } \\
\text { juego: necesidad, } \\
\text { artey derecho } \\
\text { Bally. El juego } \\
\text { como expresión } \\
\text { de libertad }\end{array}$ & $\begin{array}{l}\text { Total: } 2 \text { libros } \\
\text { Ambrosini. Del } \\
\text { monstruo al } \\
\text { estratega: éticay } \\
\text { juegos } \\
\text { Hintikka. Lógica, } \\
\text { juegos de lenguaje } \\
\text { e información: } \\
\text { temas kantianos de } \\
\text { filosofia lógica }\end{array}$ & \multicolumn{2}{|c|}{$\begin{array}{l}\text { Total: } 1 \text { libro } \\
\text { Scheines. Juguetes y jugadores }\end{array}$} \\
\hline $\begin{array}{c}\text { SOCIALES } \\
9 \text { libros en total }\end{array}$ & $\begin{array}{l}\text { Total: } 4 \text { libros } \\
\text {-Maturana y } \\
\text { Verden. Amor y } \\
\text { juego: } \\
\text { fundamentos } \\
\text { olvidados de lo } \\
\text { humano, desde el } \\
\text { patriarcado a la } \\
\text { democracia } \\
\text {-Matus. Teoría } \\
\text { del juego social } \\
\text {-Oszlak. Estado y } \\
\text { sociedad: las } \\
\text { nuevas reglas del } \\
\text { juegoran } \\
\text {-Fingermann. El } \\
\text { juego y sus } \\
\text { proyecciones } \\
\text { sociales }\end{array}$ & $\begin{array}{l}\text { Total: } 5 \text { libros } \\
\text {-Shubik. Teoría de } \\
\text { los juegos en las } \\
\text { ciencias sociales } \\
\text {-Levi y otros. } \\
\text { Juegos de escalas } \\
\text {-Braudel. Los } \\
\text { juegos } \\
\text { intercambio del } \\
\text {-Caillois. } \\
\text { juegos yos } \\
\text { hombres: los } \\
\text { máscara y el } \\
\text { vértigo. } \\
\text {-Caillois. Teoría de } \\
\text { los juegos. } \\
\text {-Berne. Juegos en } \\
\text { que participamos: } \\
\text { sicología de las } \\
\text { relaciones } \\
\text { humanas }\end{array}$ & 1 & \\
\hline $\begin{array}{c}\text { HISTORIA } \\
8 \text { libros en total }\end{array}$ & & $\begin{array}{l}\text { Total: } 6 \text { libros } \\
\text {-Lopez Cantos. } \\
\text { Juegos, fiestas y } \\
\text { diversiones en la } \\
\text { Amércia Española } \\
\text {-Paez. Del } \\
\text { truquiflor a la } \\
\text { rayuela: panorama } \\
\text { de los juegos y } \\
\text { entretenimientos } \\
\text { argentinos } \\
\text {-Gentile. } \\
\text { HuaccaMuchay. } \\
\text { Religión indigena: } \\
\text { religión, creencias, } \\
\text { juegos } \\
\text {-Duran. } \\
\text { AmuyuKudehue: } \\
\text { juegos para seguir } \\
\text { jugando y otros. } \\
\text {-Pavía y } \\
\text { Juegos que vienen } \\
\text { de antes y otros. } \\
\text {-Lonardi y florales } \\
\text { Juegos foles } \\
\text { nacionales }\end{array}$ & \begin{tabular}{lr|} 
Total: 2 libros \\
-Pellegrinelli. \\
Diccionario \\
de juguetes \\
argentinos: \\
infancia, \\
industria $\quad y$ \\
educación \\
l880-1965 \\
-AAVV. \\
Juegos r y \\
juguetes que \\
recorren el \\
tiempo
\end{tabular} & \\
\hline
\end{tabular}




\begin{tabular}{|c|c|c|}
\hline $\begin{array}{l}\text { ECONÓMICAS } \\
14 \text { libros en total }\end{array}$ & $\begin{array}{l}\text { Total: } 4 \text { libros } \\
\text {-Drovetta. } \\
\text { Administración de } \\
\text { complejidades: un } \\
\text { juego de nuevas } \\
\text { variables } \\
\text {-Green. ra } \text { La } \\
\text { dirección ante } \\
\text { situaciones de } \\
\text { crisis: cuando la } \\
\text { imagen de la } \\
\text { empresa está en } \\
\text { juego } \\
\text {-Sturzenegger. La } \\
\text { economía de los } \\
\text { argentinos: reglas } \\
\text { dejuego para una } \\
\text { sociedadpróspera } \\
\text { yjusta } \\
\text {-Aliber. El juego } \\
\text { internacional del } \\
\text { dinero. }\end{array}$ & $\begin{array}{l}\text { Total: } 10 \text { libros } \\
\text {-Gibbons. Un } \\
\text { primer curso de } \\
\text { teoría de juegos } \\
\text {-Mero y Laszlo. } \\
\text { Los azares de la } \\
\text { razón: fragilidad } \\
\text { humana, cálculos } \\
\text { morales y teoría de } \\
\text { juegos } \\
\text {-Perez Navarro y } \\
\text { otros. Teoría de } \\
\text { juegos } \\
\text {-Kreps. Teoría de } \\
\text { juegos } \\
\text { modelación } \\
\text { económica } \\
\text {-Vega Redondo. } \\
\text { Economiayjuegos. } \\
\text {-SanchezSanchez. } \\
\text { Introducción a la } \\
\text { matemática de los } \\
\text { juegos } \\
\text {-McKinsey- } \\
\text { Introducción a la } \\
\text { teoría matemática } \\
\text { de los juegos } \\
\text {-Gardner. Juegos } \\
\text { para empresarios y } \\
\text { economistas }\end{array}$ \\
\hline $\begin{array}{c}\text { HUMANIDADES } \\
1 \\
\text { deporte- } \\
\text { recreación- EF } \\
30 \text { libros en total }\end{array}$ & 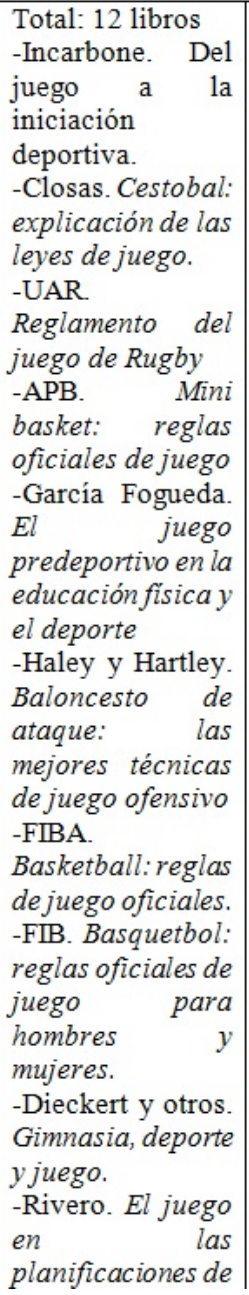 & $\begin{array}{l}\text { Total: } 18 \text { libros } \\
\text {-LavegaBurgues. } \\
\text { Juegos y deportes } \\
\text { populares } \\
\text { tradicionales } \\
\text {-Martin. Juegos y } \\
\text { recreación } \\
\text { deportiva en el } \\
\text { agua } \\
\text {-Pavey. Juegos de } \\
\text { expresión plástica } \\
\text {-Gamboa. Juegos } \\
\text { para campamentos } \\
\text {-SeguraRius. 1009 } \\
\text { ejercicios y juegos } \\
\text { de futbol } \\
\text {-DNEF. } \\
\text { Actividades fisicas } \\
\text { y juegos } \\
\text {-Hernández } \\
\text { Moreno y otros. } \\
\text { Catálogo de los } \\
\text { deportes y juegos } \\
\text { motores } \\
\text { tradicionales } \\
\text { canarios de adultos } \\
\text {-Jackman. Técnicas } \\
\text { y juegos } \\
\text { gimnásticos } \\
\text {-Cebolla Lopez. } \\
\text { Juegos con pelota. } \\
\text {-Omeñaca y otros. } \\
\text { Juegos } \\
\text { cooperativos y EF. } \\
\text {-DevisDevis } \\
\text { Peiro-Nuevas } \\
\text { perspectivas } \\
\text { curriculares n EF: }\end{array}$ \\
\hline
\end{tabular}




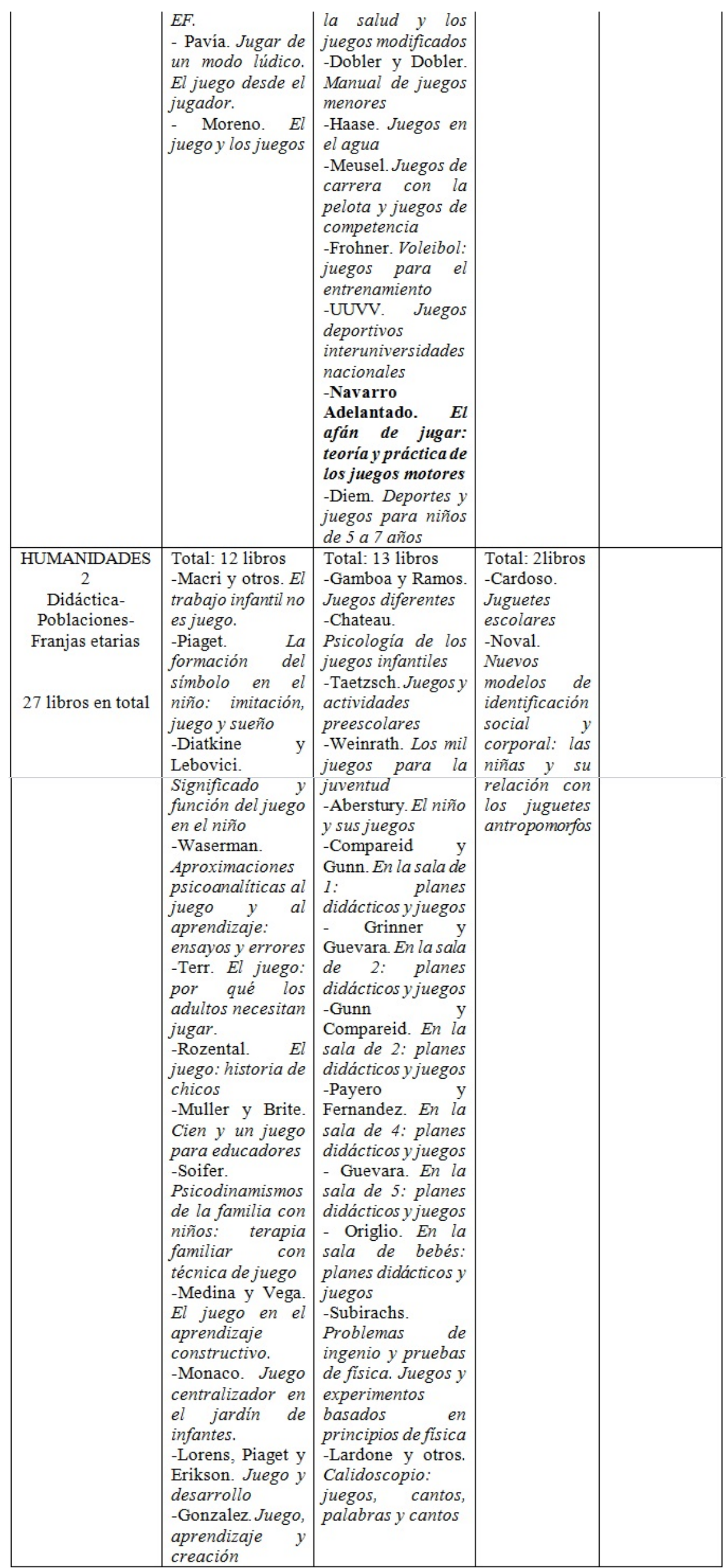


Se puede observar que más del cincuenta por ciento de los libros se corresponden al campo de las humanidades, donde las distinciones no son tan nítidas, puesto que algunos libros pueden muy bien formar parte de, al menos, dos grandes grupos. Por ejemplo, el libro Juegos y deportes populares y tradicionales está incluido en Humanidades 1 (deportes, recreación, educación física) pero puede estar al mismo tiempo en historia. Juego y juegos resultaron ser las palabras más utilizadas en los libros encontrados. Juguetes y jugadores solo se registraron en libros que impactan predominantemente en filosofía e historia. Y es que las humanidades constituyen un campo que dialoga con la historia, con las ciencias sociales, con la filosofía. Dice Ortíz (2011), que la filosofía se vale de saberes regionales, que conforman el amplio espectro de las ciencias humanas y sociales, para situar históricamente el acto de aprehensión, y para atender a la simple y antigua pregunta por el sentido, cuestión silenciada en la conceptualización científica del ser humano.

Cobran sentido así las palabras de Foucault (2017, p. 378) al decir que, "es inútil decir que las 'ciencias humanas' son falsas ciencias; no son ciencias en modo alguno; la configuración que define su positividad y las enraíza en la episteme moderna las pone, al mismo tiempo, fuera del estado de ser de las ciencias".

Si se repara en el universo de palabras mencionadas en los títulos incluidos en cada campo de conocimiento, se puede reconocer cierta afinidad que hasta puede resultar interesante analizar clarificaciones conceptuales. Así en la fila del campo de la filosofía se encuentran palabras como: reglas, posible, realidad, necesidad, arte, derecho, ética, monstruo, estratega, lenguaje, lógica, temas kantianos. En la fila de sociales se encuentran palabras como amor, patriarcado, democracia, estado, sociedad, reglas, proyecciones, teoría, escalas, intercambio, hombres, máscaras, vértigo. En la fila de historia: fiestas, diversiones, América española, truquiflor, rayuela, entretenimientos, religión indígena, creencias, infancia, industria, educación, tiempo. En la fila de económicas encontramos palabras como administración, complejidades, variables, crisis, empresa, reglas, economía, dinero, fragilidad, cálculos, modelación, matemática, empresarios y economistas. La fila de Humanidades 1 (deportes, recreación y educación física) reúne la mayor cantidad de libros. Allí se puede leer el juego en relación a leyes, reglamento, rugby, mini básquet, voleibol, futbol, competencia, ataque, técnicas, gimnasia, deporte, educación física, planificaciones; también en relación a lúdico, recreación, agua, plástica, campamentos, ejercicios, pelota, catálogo, actividades físicas, teoría, práctica, adulto. En la fila de Humanidades 2 (franjas etarias, poblaciones) se encuentran palabras como: trabajo, símbolo, imitación, sueño, significado, niño, aprendizaje, ensayos, errores, adultos, historia, educadores, familia, terapia, técnica, jardín de infantes, desarrollo, creación, juventud, planes didácticos, ingenio, pruebas, experimentos, física, cantos, palabras, identificación, niñas.

En los libros alojados en la biblioteca de la universidad tomada como caso en este estudio se puede identificar distintos grados de formalización de los estudios a los que cada libro remite. Nueve libros incluyen en su título la palabra teoría. Cinco libros incluidos en el apartado de Ciencias económicas dicen Teoría de Juegos, y tres libros cuyo título incluye la palabra teoría se encuentran en el apartado de Ciencias Sociales. De ellos, uno es la aplicación de la teoría matemática de juegos al campo de las ciencias sociales (de modo que podría perfectamente estar incluido en el apartado de Ciencias Económicas), otro remite a la teoría del juego social, de modo que el juego es utilizado como una metáfora para la explicación de la dinámica social; y el tercero, de Roger Caillois que presenta la Teoría de los juegos que toma como punto de partida el aporte del filósofo historiador holandés Johan Huizinga (2000). Finalmente, en el apartado de Humanidades 1 aportes al deporte, la recreación y la educación física se identifica un libro Teoría y práctica de los juegos motores que adhiere a la praxiologíamotriz o ciencia de la acción motriz presentada por Parlebas en la década del ‘60. 
Entonces, en la producción académica sobre juego disponible en la biblioteca seleccionada son dichas al menos tres teorías de juego: Teoría de Juegos, Teoría de los Juegos y Teoría de Juegos motores. Y aunque sería dable preguntar, ¿cuáles son los hechos académicos que colocaron la piedra angular de estas teorías?, es necesario reconocer que la retórica científica sobre juego no se agota en la descripción de estas tres teorías. De hecho, el grupo de libros incluidos en humanidades 2 aportes a franjas etarias, poblaciones, aprendizaje, parten de reconocer como antecedentes teóricos los aportes de Piaget. O bien, en el grupo de libros incluidos en el apartado de filosofía se puede identificar a Winnicot, y con él, el campo psicoanalítico. Es por ello que se avanzó en la búsqueda de los hechos académicos del siglo XX que colocaron el juego como objeto de estudio. Dice Foucault (2017, p. 371) que "el análisis de las discontinuidades busca más bien hacer surgir la coherencia interna de los sistemas significantes, la especificidad de los conjuntos de reglas y el carácter de decisión que toman, en relación con lo que han de reglamentar, la emergencia de la norma por debajo de las oscilaciones funcionales".

\section{Hechos ACADÉMICOS QUE POSICIONARON EL JUEGO COMO OBJETO DE ESTUDIO}

A pesar de estar presente en escritos desde la antigüedad (en Platón, por ejemplo), el juego hace su aparición como tema de interés en el campo académico a comienzos del siglo XIX. Seguramente las Cartas sobre la educación estética del hombre escritas por Schiller fines del siglo XVIII, editadas por Körner (con quien mantuvo el diálogo por carta) a comienzos del siglo XIX, La educación del hombre libro escrito por Froebel a comienzos del siglo XIX y los registros antropológicos de Culin de fines del siglo XIX han sido lecturas que facilitaron la aparición del juego en la escena académica. Sin embargo, el juego se consagra como objeto de estudio a comienzos del siglo XX. Al menos cinco hechos del siglo XX parecieran tener incidencia al respecto.

La creación del Instituto Jean-Jacques Rousseau en la Universidad de Ginebra en 1912, por Claparáde puede ser reconocido como un primer hecho que posicionó como objeto al juego en la producción académica. Este instituto dio lugar al Instituto de Ciencias de la Educación, donde se realizaban investigaciones de psicología educativa dirigidas por Piaget desde 1921 hasta que fue nombrado director del instituto en 1935. Es aquí donde consiguen reconocimiento un conjunto de producciones provenientes de los campos de la biología (Spencer, Groos, Lazarus) y de la psicología (Hall, Claparade, Freud, Buytendijk), que serán reconocidas como teorías clásicas del juego a pesar de que su objeto no fuera el juego sino el desarrollo humano. Spencer será un autor que arrastra al campo educativo incidencias de la filosofía de la evolución y tendrá su arrastre al campo de la educación física (Galak, 2016). Este ha sido el discurso dominante sobre juego en el campo educativo que lo colocó en el lugar de instrumento o medio para enseñanza de otros saberes.

En 1920, Freud escribe El fort da en Más allá del principio del placer donde se dedica al funcionamiento del aparato anímico en los juegos infantiles a partir de un caso. Esta descripción coloca el juego en la consecución de placer y, aunque servirá de base para la producción psicoanalítica sobre juego, gana visibilidad en la escena académica con la publicación de Realidad y juego de Winnicot en la década del '60.

El tercer hecho es la conferencia central de Huizinga en la Universidad de Leiden (Holanda), luego de la cual publica el Homo ludens (1938). Este será el hecho académico de mayor relevancia puesto que es el primer libro que tematiza el juego, enraizándolo a la cultura. Este libro servirá de base a Caillois para 
escribir la Teoría de los Juegos ${ }^{9}$. Este discurso sobre el juego expandirá su potencia en distintas producciones académicas del campo de la filosofía (entre las cuales se destaca la tesis doctoral de Eugen Fink) expandiendo su alcance al campo de las humanidades y sociales a lo largo del siglo XX. Este discurso marca una distinción en las producciones antropológicas de fines de siglo XIX (Culin, entre otros) que, hasta ese entonces, se interesaban por los mensajes que los juegos brindan sobre la sociedad y que fueron condensadas en la creación de la Asociación Antropológica para el Estudio del Juego acontecida en 1974 (Enriz, 2011). La Declaración de Ginebra de 1924, primer tratado internacional sobre los derechos del niño aprobado por la Liga de las Naciones fue un hecho político que provocó la aparición de instrumentos legales (como la Convención de los Derechos del Niño, y la Ley del Deporte) y una serie de organizaciones no gubernamentales dedicadas al juego que nacen en segunda mitad del siglo XX. Entre ellos, la Asociación Internacional por el Derecho del Niño a Jugar (IPA) que publica el libro El juego: necesidad, arte y derecho, y el Instituto para la Investigación y Pedagogía del Juego que tiene una sede en Buenos Aires, es dirigido por la Dra. Regina Öfele quien compiló el libro Homo Ludens. El hombre juega 1. En esta obra publica un artículo Brian Sutton Smith, un autor que se desempeñó como presidente de la Asociación Antropológica para el Estudio del Juego y también de la Asociación Americana de Psicología.

En 1944 tiene lugar un cuarto hecho académico que coloca el juego como objeto: la publicación de una serie de estudios matemáticos que inspirarán la Teoría de Juegos y comportamiento económico de von Neumann y Morgenstern. En ellos pudo haber tenido influencia el pensamiento de Frobenius, matemático alemán referenciado en la obra de Huizinga.

Un quinto hecho académico acontece en 1968,Parlebaspublica la Educación Física estructural que sentará las bases de una propuesta teórica con pretensiones científicas, a la que llamará praxiología motríz o ciencia de la acción motríz. Toma como eje de análisis a los juegos y los deportes ("juegos deportivos no institucionalizados" y "juegos deportivos institucionalizados", en su propia terminología) y los entiende como prácticas culturales diferentes. Las producciones de Parlebas otorgan fundamentación científica a los juegos desde un análisis estructuralista factible de ser matematizado. Su aporte radica entender la motricidad como fenómeno social, como un hecho de comunicación, de interacción y de intercambio. Aunque provoca movilización y discusión en el campo de la educación física y genera una copiosa producción académica en Europa, la praxiología motríz francesa no consigue gran adhesión en Argentina y Latinoamérica (Saraví, 2006).

Entonces ahora la pregunta es, ¿qué juego es el que pronuncian las producciones académicas que lo colocaron como objeto de estudio?, ¿cuáles han sido las lecturas de los autores que presentaron el juego en la academia?, ¿cuándo fue que se empezó a creer que el juego es una práctica que se aprende sin enseñanza?, ¿cuándo se empezó a creer que el juego carece de teoría por estar supeditado a las reacciones instintivas?

Aun cuando el escenario teórico del juego se presenta amplio y complejo, es posible reconstruir en él la idea de juego en tanto práctica autotélica asociada al disfrute, en tanto práctica popular reconocida tradicionalmente como juego (que va desde las expresiones lúdicas más simples y rudimentarias hasta la organización voluptuosa y exuberante del espectáculo y la previsión, u objetos extravagantes), presente en la vida cotidiana de las sociedades, en las distintas expresiones culturales (obras de arte, pinturas, canciones, cuentos, historias, juguetes, tableros), en los espacios públicos abiertos (como plazas, patios, parques y paseos) y en espacios privados (casas, escuelas, clínicas, centros, peloteros, clubes, gimnasios). 
Los juegos, juguetes, tiempos y lugares para jugar han participado y participan en el proceso de regulación y control de los cuerpos en las sociedades modernas, proceso en el que la educación, y con ella la educación física, han hecho y hacen lo suyo. Una incipiente y copiosa producción académica latinoamericana en el campo de la educación de los cuerpos (Scharadrosky y Gleyse, 2013, Galak y Varea, 2013; Galak, 2016, Galak y Gambarotta, 2015; Crisorio, 2017; Almeida y Eussen, 2018; Uribe, Gallo y Fernandez Vaz, 2017) invita a desnaturalizarlo, cuestión presente en los estudios de Pavía $(2006,2011)$ que desde 1994 lleva adelante en la escuela, los de Sarlé (2001) en jardines de infantes, escritos de Centurión (2006) en la formación universitaria, y los de Rivero $(2011,2012)$ en la formación docente en educación física.

Esto justifica el análisis crítico de prácticas y discursos que han colaborado en la construcción, institucionalización (en la escuela, clubes, peloteros) y sostenimiento de juegos y juguetes que aíslan y aquietan (pantallas), que destacan al habilidoso (y/o denigran al inhábil o novato), que distinguen acciones por género, juegos en los que algunos participantes no consiguen jugar, disfrutar, divertirse.

El interés está puesto en las prácticas, en los juegos de la plaza/parque/paseos, el patio de la escuela, el club, en las propuestas de juego en talleres y clases de educación corporal en la escuela y fuera de ella (colonia de vacaciones, centros de jubilados, gimnasios, centros de rehabilitación), lugares de encuentro. Los momentos de cuidado de personas, de prácticas recreativas y de educación sistemática han contribuido a instalar prácticas, objetos, actividades, lugares y modalidades de juego que requieren revisión para sacar del letargo las problemáticas que se anudan entre el juego y la competencia, juego y violencia, juego y soledad, juego y quietud, los juegos y la construcción de género, temáticas acuciantes del siglo XXI.

\section{Bibliografía}

Almeida, F. Q., y Eusse, K. L. G. (2018). Educação Corporal: uma análise comparada entre a Colômbia e a Argentina. En Educación Física y Ciencia, 20(1), e044. https://doi.org/10.24215/23142561e044

Bourdieu, P; Chamboredon, J., y Passeron, J. (2011).El oficio del sociólogo. Presupuestos epistemológicos. Buenos Aires: Siglo XXI.

Bourdieu, P., y Wacquant, L. (2005). Una invitación a la sociología reflexiva. Buenos Aires: Siglo XXI.

Centurión, S. (2006). El juego como propuesta académica. En I. Rivero y M. Ducart (2017). El juego en la formación docente: acerca del juego como recurso.(pp. 26-34). Río Cuarto: UniRío. Recuperado de: https://www.unrc.edu. $\mathrm{ar} / \mathrm{unrc} / \mathrm{comunicacion/editorial/repositorio/978-987-688-246-0.pdf}$

Crisorio, R. (2003). Educación Física e identidad: conocimiento, saber y verdad. En Bracht V. y Crisorio, R. (Coord.) (2003). La Educación Física en Argentina y en Brasil. Identidad, desafios y perspectivas (pp.21-38). La Plata: Al margen.

Crisorio, R. (2015). Parte I. El objeto de estudio. En Crisorio, R.; Rocha Bidegain, L.; Lescano, A. (Coord.) (2015). Ideas para pensar la educación del cuerpo(Pp. 8-39). La Plata: Edulp. Recuperado de: http://sedici.unlp.edu.ar

Crisorio, R., (2017). El cuerpo, entre Homero y Platón. Buenos Aires: Biblos.

Enriz, N. (2011). Antropología y juego: apuntes para la reflexión. Cuadernos de Antropología Social, (34), 93-114. Disponible en: https://www.redalyc.org/articulo.oa?id=180922374005

Foucault, M. (2017). Las palabras y las cosas. Una arqueologia de las ciencias humanas. Buenos Aires: Siglo XXI. 
Galak, E. y Varea. V. (2013). Cuerpo y educación física. Perspectivas latinoamericanas para pensar la educación de los cuerpos. Buenos Aires: Biblos.

Galak E. y Gambarotta, E.(comp.) (2015). Cuerpo, educación, politica: tensiones epistémicas, históricas y prácticas. Buenos Aires: Biblos.

Galak, E. (2016). Educar los cuerpos al servicio de la politica. Cultura fisica, higienismo, raza y engenesia en Argentina y Brasil.Buenos Aires: Biblos.

Huizinga, J. (2000). Homo ludens. Madrid: Alianza.

Larrosa, J. (2000). Pedagogía profana. Estudios sobre lenguaje, subjetividad, formación. Buenos Aires: Novedades educativas.

Montilla Peña, L.(2012) Análisis bibliométrico sobre la producción científica archivística en la Red de Revistas Científicas de América Latina y el Caribe (Redalyc) durante el período 2001-2011. Biblios, (48), 1-11. Recuperado de: https://www.redalyc.org/articulo.oa?id=16126403006

Ortiz, G.(2011). Tiempos indigentes. Sobre la religión, la educación y la pregunta por el sentido. Córdoba: EDUCC.

Pavía, V. (Coord.)(2006). Jugar de un modo lúdico. El juego desde la perspectiva del jugador. Buenos Aires: Novedades Educativas.

Pavía, V. (Coord.) (2011). Formas del juego y modos de jugar. Secuencias de Actividades Lúdicas. Santa Fé: AMSAFE.

Rivero, I. (2006). El uso del lenguaje en el juego infantil. En Pavía, V (Coord.)(2006). Jugar de un modo lúdico. El juego desde la perspectiva del jugador (Pp. 55-68). Buenos Aires: Novedades Educativas.

Rivero, I. (2009). La acción de jugar. El desafío de develar el lenguaje del juego. En Chaverra Fernandez, B. (Coord.) (2009), Juego y deporte: reflexiones conceptuales hacia la inclusión. (Pp. 35-46). Medellín: Funámbulos.

Rivero, I. (2011). El juego en las planificaciones de Educación Física. Intencionalidad educativa y prácticas docentes. Buenos Aires: Novedades educativas.

Rivero, I. (2012). El juego desde la perspectiva de los jugadores: Una investigación para la didáctica del jugar en educación física. Tesis Doctorado. La Plata: Memorias Académicas FaHCE- UNLP. Recuperado de: http://w ww.memoria.fahce.unlp.edu.ar/tesis/te.901/te.901.pdf

Rivero, I.; Gilleta, G. y Limbrici Dagfal, M. (2019). ¿Por qué callar lo que se hace? El juego en aulas universitarias innovadoras. En Bono, A. y Aguilera, S. (Comp.) (2019). Notas sobre investigación en Humanidades (pp. 129-142). Río Cuarto: UniRío. Recuperado de: http://www.unirioeditora.com.ar/wp-content/uploads/2019/ 09/978-987-688-353-5.pdf

Saravi, J. (2006) Praxiología motriz: Un debate pendiente. Educación Física y Ciencia, (9), 103-117. Recuperado de: https://www.efyc.fahce.unlp.edu.ar

Sarlé, P. (2001). Juego y aprendizaje escolar. Los rasgos del juego en la educación infantil. Buenos Aires: Novedades Educativas.

Scharagrodsky, P., y Gleyse, J. (2013). El Dr. Enrique Romero Brest, las visitas a instituciones europeas de formación y el Congreso de Educación Física realizado en 1913 como indicadores de la globalización y la nacionalización de la 'cultura física'. Staps, 2(100), 89-107.

Uribe, I.; Gallo, L. y Fernandez Vaz, A. (2017). Trazos de una educación hedonista. Movimento, Porto Alegre, 23(1), 339-350.

\section{Notas}

1 Este cambio fue posible gracias a la participación de nuestro equipo de juego en la Red NEIES, pues el Dr. Crisorio, director de la Red, dirige mi beca.

2 Cagigal, J. 1979. Cultura intelectual y culturafisica. Kapeluz. Buenos Aires. Gruppe en Pedráz, M. 1988. Teoría pedagógica de la Educación Fisica. Madrid. España. Gymnos Editorial. Seybold, A. 1974. Principios pedagógicos en la educación física. Kapeluz. Buenos Aires. 
3 Sobre la colocación del juego como objeto de enseñanza en la Educación Física, se puede visitar Centurión, S. El juego como propuesta académica. En Rivero, I. y Ducart, M. (Comp.) 2017. El juego en la formación docente. Acerca del juego como recurso. Unirío: Río Cuarto. www.unrc.edu.ar/unrc/comunicacion/editorial.

También se puede recuperar Rivero, I. 2019. Trazos de la construcción del objeto juego. Actas Congreso de EF y Ciencias http://congresoeducacionfisica.fahce.unlp.edu.ar. Allí, se presenta el análisis del impacto que tuvieron en el estudio y reconocimiento del juego en la educación física que venimos siendo, es decir, la educación de los cuerpos, el Congreso Nacional de Educación Física de Villa Giardino, el libro Juegos que vienen de antes. Incorporando el patio a la escuela, de Víctor Pavía y el libro Educación Física. De la alegría de lo lúdico a la opresión del rendimiento de Silvino Santin.

4 Formación de grado en Educación Física que comienza para esos años en Argentina.

5 La incorporación del juego en los NAP es un hecho que merece especial mención, puesto que implicó la formalización de un contenido a enseñar en las escuelas.Su análisis excede las pretensiones de este escrito pero entra en la agenda de tareas de la investigación en curso.

6 De hecho, en el análisis crítico de la Educación Física del momento que el autor hace en el libro Educación física y aprendizaje social, se pueden apreciar pasajes de alto valor para la colocación del juego como objeto de estudio. Por citar un ejemplo, "en la perspectiva autónoma se acentúa la dimensión lúdica del ser humano" (Bracht, 1992, p. 46). Este libro fue lectura obligatoria en Sociología de la Educación en las siete sedes en que se dictó la licenciatura de Educación Física de la UNRC: Río Cuarto ( 5 cohortes), Córdoba (3 cohortes), Villa María, Bell Ville, Rafaela, Villa Mercedes, Villa Dolores. (Agradezco cortesía del profesor Carlos Valentinuzzi, coordinador de la carrera, además de escribir y gestionar su aprobación académica). Por la alta incidencia que las ideas de este autor han tenido en la institución seleccionada para el estudio, su estudio será presentado en otro escrito

7 El ejercicio que aquí se propone es el de ampliar la lente para identificar los campos de conocimiento que cuentan con autores que colocan al juego en el título de su obra.De hecho, de los textos presentados en los apartados anteriores y que justifican la línea de estudio del Juego desde los Jugadores,que contribuye a pensar el juego desde y para la educación de los cuerpos, solo se encuentran aquellos que mencionan el juego en el título.

8 En el grado: Conocimiento y juego en la formación de profesores de Educación Física, Juego, Juego dramático y Seminario Juego en la formación de profesoras y licenciadas en Educación Inicial. En el posgrado: un curso de Teoría de Juegos (Ciencias Económicas) y catorce cursos independientes pero interrelacionados en dos Diplomaturas Superiores, una en Juego y Educación Física; y otra en Juego y Prácticas Corporales (Ciencias Humanas).

9 Para mayor información sobre el contexto de producción de Huizinga y Caillois ver Rivero, I. El juego desde los jugadores. Huellas en Huizinga y Caillois. En Enrahonar. Quaderns de Filosofia 56, 2016. Pág. 49-63. 